\section{D) Check for updates}

Cite this: Nanoscale, 2020, 12, 7309

\title{
Near-field mechanism of the enhanced broadband magneto-optical activity of hybrid Au loaded $\mathrm{Bi}$ : YIG†
}

\author{
Spiridon D. Pappas, (D) *a Philipp Lang, ${ }^{a}$ Tobias Eul, ${ }^{a}$ Michael Hartelt, ${ }^{a}$ \\ Antonio García-Martín, (D b Burkard Hillebrands, ${ }^{a}$ Martin Aeschlimann (D) a and \\ Evangelos Th. Papaioannou (D) *a,c
}

\begin{abstract}
We unravel the underlying near-field mechanism of the enhancement of the magneto-optical activity of bismuth-substituted yttrium iron garnet films (Bi:YIG) loaded with gold nanoparticles. The experimental results show that the embedded gold nanoparticles lead to a broadband enhancement of the magnetooptical activity with respect to the activity of the bare Bi:YIG films. Full vectorial near- and far-field simulations demonstrate that this broadband enhancement is the result of a magneto-optically enabled crosstalking of orthogonal localized plasmon resonances. Our results pave the way to the on-demand design of the magneto-optical properties of hybrid magneto-plasmonic circuitry.
\end{abstract}

Received 8th January 2020,

DOI: $10.1039 /$ dOnr00198h

rsc.li/nanoscale

\section{Introduction}

The field of magneto-plasmonics has attracted a lot of scientific interest, both for its importance in potential technological applications, and for its fundamental scientific relevance. ${ }^{1-4}$ Towards these directions, various ideas like using external magnetic fields to control the dispersion relation of plasmonic resonances (active magneto-plasmonics), ${ }^{5}$ or using plasmonic resonances for spin current generation in adjacent magnetic insulators, ${ }^{6,7}$ and the implementation of a magneto-plasmonic interferometer, ${ }^{8}$ have been realized and explored.

Magneto-optical studies on magneto-plasmonic nanostructures, composed of magnetic and/or plasmonic materials, have revealed new exciting effects: nanopatterned hybrid heterostructures, ${ }^{9-16}$ pure ferromagnetic films, ${ }^{17-24}$ and noble metal/magnetic dielectric systems ${ }^{25,26}$ exhibit plasmon-induced enhancement of their magneto-optical activity. Recently, the correlation of near- and far-field effects of a patterned magnetoplasmonic array has been shown with the aid of Photoemission Electron Microscopy (PEEM), ${ }^{27}$ paving the way for tailoring the magneto-optical response of these systems: the spatial distribution of the polarization- and energy-dependent electric near-

\footnotetext{
${ }^{a}$ Department of Physics and Research Center OPTIMAS, University of Kaiserslautern, 67663 Kaiserslautern, Germany.E-mail: pappas@rhrk.uni-kl.de

${ }^{b}$ Instituto de Micro y Nanotecnología IMN-CNM, CSIC, CEI UAM+CSIC, Isaac Newton 8, E-28760 Tres Cantos, Madrid, Spain

${ }^{c}$ Institute of Physics, Martin-Luther University Halle-Wittenberg, Von-DanckelmannPlatz 3, 06120 Halle, Germany.E-mail: evangelos.papaioannou@physik.uni-halle.de $\dagger$ Electronic supplementary information (ESI) available. See DOI: 10.1039/ d0nr00198h
}

field of the propagating plasmon polaritons has been connected to the enhancement of the magneto-optical Kerr effect. As a further matter, the nature of the plasmonic resonances (localized or propagating surface plasmons ${ }^{28,29}$ ) can alter differently the response of the magneto-optically active material in a magneto-plasmonic structure. From the wider family of the hybrid magneto-plasmonic structures, ${ }^{14-16,25,30,31}$ the system composed of ferrimagnetic dielectric layers of bismuth-substituted yttrium iron garnet (Bi:YIG) with embedded $\mathrm{Au}$ nanoparticles (AuNPs) that support localized plasmon resonances (LPRs), has attracted little attention for its importance. ${ }^{26,32,33}$ The underlying near-field mechanism is not clarified yet, while there is lack of studies on the plasmon-induced magnetooptical response in the Longitudinal Magneto-optical Kerr Effect (L-MOKE) configuration.

In this paper, we explore the microscopic origin of the magneto-plasmonic coupling in bismuth-substituted yttrium iron garnet films (Bi:YIG), loaded with self-assembled $\mathrm{Au}$ nanoparticles (AuNPs). We can unambiguously prove that the features in the enhanced magneto-optical activity are the result of two coupled orthogonal LPRs. The coupling is mediated by the magneto-optical activity of the underlying Bi:YIG matrix, for both polarization states of incident light.

\section{Results and discussion}

\section{Hybrid Au loaded Bi:YIG films}

In Fig. 1(a), the structure of the investigated hybrid sample (Bi:YIG/AuNPs) is presented. Scanning electron microscopy 
(a)

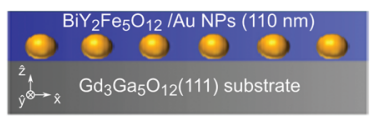

(c)

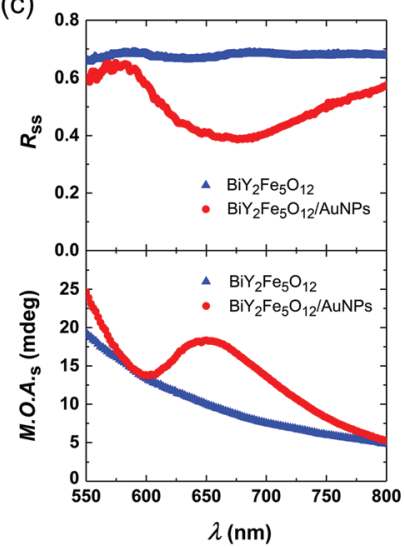

(b)
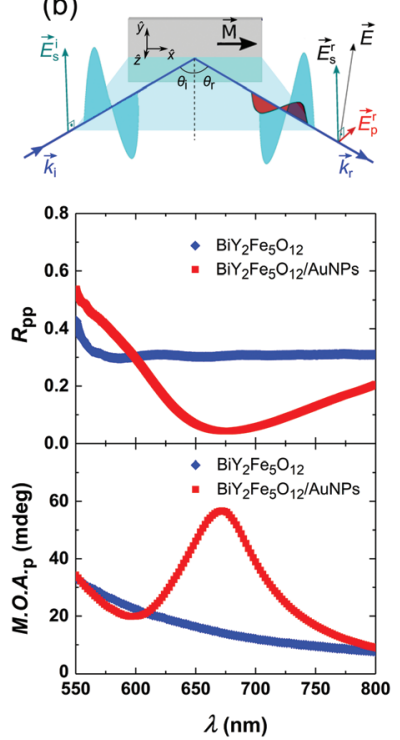

Fig. 1 (a) Schematic cross section of the sample used in the present study. (b) Scheme of the longitudinal MOKE configuration, used to record the Kerr angle of the $\mathrm{BiY}_{2} \mathrm{Fe}_{5} \mathrm{O}_{12}$ /(AuNPs) samples. (c) Far-field measurements: (Top graphs) Experimental $R_{\mathrm{ss}}$ and $R_{\mathrm{pp}}$ reflectance plots as a function of wavelength for the $\mathrm{BiY}_{2} \mathrm{Fe}_{5} \mathrm{O}_{12} /$ (AuNPs) samples. (Bottom graphs) Dependence of the magneto-optical activity (M.O.A.s(p)) as a function of wavelength, recorded for the $\mathrm{BiY}_{2} \mathrm{Fe}_{5} \mathrm{O}_{12} /$ (AuNPs) samples for $\mathrm{s}$ - and $\mathrm{p}$-polarized incident light. Both the reflectance as well as the M.O.A. spectra have been recorded for angle of incidence $\theta_{\mathrm{i}}=55$ deg.

(SEM) images of the sample (see ref. 6) reveal that the AuNPs are randomly distributed close to the interface between Bi:YIG and gadolinium gallium garnet (GGG), and that they are embedded in the Bi:YIG film. Furthermore, they have the shape of oblate spheroids, with an in-plane diameter ranging from 30 to $90 \mathrm{~nm}$ and a height ranging from 20 to $50 \mathrm{~nm}$. The optical properties of this sort of system are mainly determined by localized plasmon resonances (LPRs), rather than by geometrical-lattice resonances. A second sample, having exactly the same structure, but containing no AuNPs $\left(\mathrm{BiY}_{2} \mathrm{Fe}_{5} \mathrm{O}_{12}\right)$, was used as a reference. The reader is referred to the $\mathrm{ESI} \dagger$ for further details on the fabrication of the samples.

\section{The L-MOKE configuration}

The magneto-optical response of the samples was experimentally probed in the L-MOKE configuration, which is schematically depicted in Fig. 1(b). In the L-MOKE geometry, the magnetization $(\boldsymbol{M})$ of the sample lies in the sample plane, as well as in the plane of incidence of the incident light. The incident light can either be s- or p-polarized. In Fig. 1(b) only the case of a measurement performed with s-polarized light is presented for simplicity. Superscripts $r$ and i denote the reflected and incident light, respectively. The reflected light contains a p-polarized component which causes a change in the polariz-

ation state. The polarization state of the reflected light is then given by:

$$
\chi_{\mathrm{s}}=\frac{E_{\mathrm{p}}^{\mathrm{r}}}{E_{\mathrm{s}}^{\mathrm{r}}}=\frac{r_{\mathrm{ps}}}{r_{\mathrm{ss}}} \approx \theta_{\mathrm{s}}+i \varepsilon_{\mathrm{s}}
$$

or

$$
\chi_{\mathrm{p}}=\frac{E_{\mathrm{s}}^{\mathrm{r}}}{E_{\mathrm{p}}^{\mathrm{r}}}=\frac{r_{\mathrm{sp}}}{r_{\mathrm{pp}}} \approx \theta_{\mathrm{p}}+i \varepsilon_{\mathrm{p}}
$$

where $\chi_{\mathrm{s}(\mathrm{p})}$ is the complex Kerr angle, $\theta_{\mathrm{s}(\mathrm{p})}$ is the Kerr rotation, and $\varepsilon_{\mathrm{s}(\mathrm{p})}$ is the Kerr ellipticity for $\mathrm{s}^{-}(\mathrm{p}-)$ incident polarized light. In eqn (1) \& (2) $E_{\mathrm{s}(\mathrm{p})}$ is the magnitude of the electric field for s- or p-polarization state of light, and $r_{\mathrm{ss}}, r_{\mathrm{pp}}, r_{\mathrm{ps}}$ and $r_{\mathrm{sp}}$ are the Fresnel coefficients. Optical reflectance spectroscopy has been performed for both samples, as well. The reflectance spectra were recorded by using the exact same geometry which was used for the study of the magneto-optical response, with angles $\theta_{\mathrm{i}}=\theta_{\mathrm{r}}=55 \mathrm{deg}$. For a more detailed description of the experimental techniques the reader is referred to ESI. $\dagger$

\section{Measured magneto-optical response}

In Fig. 1(c) (top graphs), we present the measured reflectance spectra for both incident s- $\left(R_{\mathrm{ss}}\right.$ plot $)$ and p-polarized $\left(R_{\mathrm{pp}}\right.$ plot $)$ light. It is apparent, that the reflectance of the sample containing no AuNPs, retains a constant value in the spectral region of 550-800 nm. In the case of the sample containing AuNPs, a broad drop in the $R_{\mathrm{ss}}$ and $R_{\mathrm{pp}}$ spectra is located in the spectral region 550-900 $\mathrm{nm}$, reaching a minimum at $\sim 675 \mathrm{~nm}$. The reflectance reduction in this spectral region, where the electronic transitions of Bi:YIG ${ }^{34,35}$ have no relevant influence, is solely attributed to localized plasmon resonances (LPRs) in the AuNPs, for sizes smaller than $\sim 100 \mathrm{~nm}$.

Our interest is particularly focused on the influence of the LPRs on the magneto-optical response in the L-MOKE configuration. The L-MOKE configuration is easy to be implemented, and therefore quite attractive for technological applications, as well. In order to obtain a direct insight into the effect, we extracted the modulus of the complex Kerr angle $\chi_{\mathrm{s}(\mathrm{p})}$, which is also called the magneto-optical activity $\left(M . O \cdot A \cdot \mathrm{s}(\mathrm{p})=\sqrt{\theta_{\mathrm{s}(\mathrm{p})^{2}+\varepsilon_{\mathrm{s}(\mathrm{p})}}}\right)$, for applied external magnetic fields sufficiently large to saturate the sample along the film in-plane direction. The spectral dependence of M.O.A. for sand p-incident polarized light is shown in the bottom graphs of Fig. 1(c) for both samples. The increased M.O.A. of Bi:YIG in the low-wavelength spectral region is attributed to the wings of the magneto-optical transitions located at photon energies of 2.8 and $3.3 \mathrm{eV}$ (442 and $378 \mathrm{~nm}$ respectively). ${ }^{35}$ It becomes apparent that the magneto-optical response of the Bi:YIG layer, for the sample containing AuNPs, is strongly modified in the broad optical region where the resonant localized surface plasmon phenomena occur. Furthermore, by observation it can be deduced that the enhancement of the M.O.A. is larger in the case of incident p-polarized light. The experimental results clearly show a significant broadband enhancement of the magneto-optical response of Bi:YIG in a spectral regime far 
from the inherent Bi:YIG magneto-optical transitions, which originates from LPRs in the hybrid sample.

\section{Near-field simulations \& analysis}

In order to gain insight into the deeper mechanism of the enhanced magneto-optical activity of the hybrid $\mathrm{BiY}_{2} \mathrm{Fe}_{5} \mathrm{O}_{12} /$ AuNPs structure, we performed numerical simulations with the aid of Computer Simulation Technology (CST) software package and analyzed both the electric near-field features, as well as the far-field magneto-optical behaviour (see $\mathrm{ESI} \dagger$ for further details on the Method). To perform these simulations, the dielectric tensor of $\mathrm{BiY}_{2} \mathrm{Fe}_{5} \mathrm{O}_{12}$ was used. For the L-MOKE geometry and the vector directions as they are defined in Fig. 1(b), the dielectric tensor of Bi:YIG has the following form:

$$
\boldsymbol{\epsilon}^{\mathrm{Bi}: \mathrm{YIG}}(\lambda)=\left(\begin{array}{ccc}
\epsilon_{x x} & 0 & 0 \\
0 & \epsilon_{y y} & \epsilon_{y z} \\
0 & \epsilon_{z y} & \epsilon_{z z}
\end{array}\right)
$$

with $\epsilon_{x x} \approx \epsilon_{y y} \approx \epsilon_{z z} \approx \epsilon(\lambda)$ and $\epsilon_{z y}=-\epsilon_{y z}=-i \epsilon(\lambda) Q$, where $Q$ is the Voigt constant. The M.O.A. of Bi:YIG originates from spinorbit (SO) coupling and thus, it is an intrinsic property of the material. The off-diagonal elements $\epsilon_{z y}$ and $\epsilon_{y z}$ of the dielectric tensor account for the effects of the SO interaction on the dielectric properties of the magneto-optically active material. The values of the diagonal and off-diagonal elements as a function of wavelength of Bi:YIG were obtained from the existing literature. ${ }^{35,36}$ The dielectric tensor values for Au and GGG were taken from existing literature, as well. ${ }^{36-39}$ The angle of incidence for the incoming planar electromagnetic wave was defined at $55 \mathrm{deg}$, in order to match with the angle of incidence which was used for the measurements (presented in Fig. 1(c)). The AuNPs are modeled as oblate spheroids with fixed values of semi-axes: $a=60 \mathrm{~nm}$ and $c=35 \mathrm{~nm}$. These values are chosen to correspond to the mean value of the nanoparticle sizes obtained from cross-sectional transmission electron microscopy (TEM) images. ${ }^{6}$

In Fig. 2(a) (left side) the maps of the spatial $y$ component $\left(E_{y}\right)$ of the local electric field $\boldsymbol{E}$ is presented at two characteristic spectral positions: $\lambda=550 \mathrm{~nm}$, and $727 \mathrm{~nm}$. In this case the incident electric field is s-polarized. The maps reveal the spectral position of the electric near-field enhancement which emanate from the localized plasmon resonances in AuNPs. As it can be observed, at $\lambda=727 \mathrm{~nm}$ a big volume of the hosting Bi:YIG is exposed to the enhanced near-field around the AuNP. In order to quantify these near-field results in a more comprehensive way, and reveal the exact spectral positions of the plasmonic resonances, we calculate the induced dipole moment in the AuNP. In the small particle limit, the dipole moment induced in the AuNP, which is embedded in the dielectric Bi: YIG, is given by the following formula: ${ }^{40,41}$

$$
\boldsymbol{p}=\epsilon_{\mathrm{o}}\left(\boldsymbol{\epsilon}^{\mathrm{Au}}-\boldsymbol{\epsilon}^{\mathrm{Bi}: \mathrm{YIG}}\right) \frac{1}{N_{\mathrm{p}}} \sum_{m} V_{m} \boldsymbol{E}_{m}
$$

where $\epsilon_{\mathrm{O}}$ is the vacuum permittivity, $\epsilon^{\mathrm{Au}}$ is the dielectric tensor of $\mathrm{Au}$, and $\boldsymbol{\epsilon}^{\mathrm{Bi} \text { :YIG }}$ is the dielectric tensor of the surrounding $\mathrm{Bi}$ : (a)

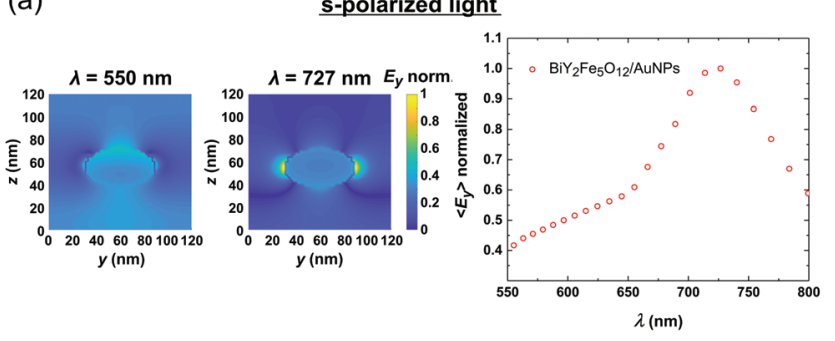

(b) p-polarized light

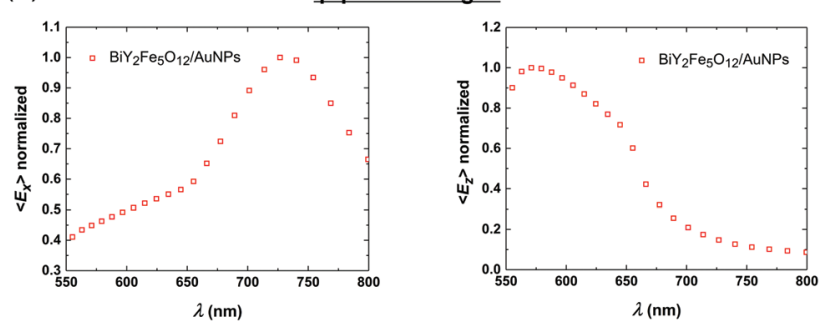

Fig. 2 Near-field simulations: (a) (Left side) Maps of the $y$ electric nearfield component $\left(E_{y}\right)$ for two different wavelength values of incident s-polarized light. The color scale has been normalized to the maximum spatial field value in each case. (Right side) Calculated $\left\langle E_{y}\right\rangle$ in the AuNP, for incident s-polarized light. The values have been calculated from the simulated $y$ electric near-field components in the Au nanoparticle. The $\left\langle E_{y}\right\rangle$ values have been normalized to the maximum value. (b) Calculated $\left\langle E_{x}\right\rangle$ and $\left\langle E_{z}\right\rangle$ in the AuNP, for $p$-polarized incident light. The values have been calculated by following the same procedure as in (a).

YIG material. $N_{\mathrm{p}}$ is the total number of the discrete mesh cells, at which each value of the electric field $\boldsymbol{E}_{m}$ is calculated numerically. $V_{m}$ is the volume of the $m^{\text {th }}$ cell of the total discretized volume. From eqn (4), it can be deduced that $\boldsymbol{p} \propto\langle\boldsymbol{E}\rangle$, where $\langle\boldsymbol{E}\rangle=\frac{1}{N_{\mathrm{p}}} \sum_{m} V_{m} \boldsymbol{E}_{m}$ is the average electric field in the AuNP. The calculated $y$ component of the averaged field $\left(\left\langle E_{y}\right\rangle\right)$ as a function of the wavelength of the incident light, in the case of s-incident light, is shown in the right hand side plot of Fig. 2(a). The results show a clear intensification of the average field along the $y$ direction, with the peak of $\left\langle E_{y}\right\rangle$ located at $727 \mathrm{~nm}$. All of the values have been normalized to the maximum value of the curve. In the case of p-polarized light, the vector of the oscillating incident electric field can be analyzed along the $x$ and the vertical $z$ direction. Therefore, in Fig. 2(b), we choose to show both $\left\langle E_{x}\right\rangle$ and $\left\langle E_{z}\right\rangle$. The plots reveal the existence of a clear intensification along the $x$ direction (where $\left\langle E_{x}\right\rangle$ is considered), as well as a clear but more feature-complicated intensification spectrum along the $z$ direction (where $\left\langle E_{z}\right\rangle$ is considered). By comparison, we can deduce that the $\left\langle E_{y}\right\rangle$ plot in the case of s-polarized incident light, and the $\left\langle E_{x}\right\rangle$ plot in the case of p-polarized incident light, have identical shapes. This can be clearly explained from the symmetry of the geometrical shape of the simulated nanoparticle on the $x y$ plane. From the $\left\langle E_{z}\right\rangle$ plot in Fig. 2(b), we can observe a field intensification close to the lower limit of the simulated spectral region. A clear peak is shown at $\lambda=575 \mathrm{~nm}$, with an extra feature at about $\lambda=625 \mathrm{~nm}$. In the case of p-polarized 
incident light, $\left\langle E_{z}\right\rangle$ becomes enhanced at a much smaller wavelength value than the component $\left\langle E_{x}\right\rangle$ does. This, can be understood by the fact that the dimension of the nanoparticle along the $z$ direction is smaller than that along the $x$ or $y$ direction. Therefore, the resonant mode along $z$ is shifted at lower wavelength values, as it is compared to the resonances along the $x$ or $y$ direction. Furthermore, the fact that the $\left\langle E_{z}\right\rangle$ curve has a more complex shape, in comparison to the $\left\langle E_{x}\right\rangle$ curve, could be explained by the geometric complexityof the oblate spheroid shape.

\section{Far-field simulations \& analysis}

Subsequently, we want to compare the near-field simulations with the obtained far-field data. Therefore, we initially calculated the reflectivity for incident s- and p-polarized light, $R_{\mathrm{ss}}$ and $R_{\mathrm{pp}}$ respectively. Additionally, the polarization conversion efficiencies $R_{\mathrm{ps}}$ and $R_{\mathrm{sp}}$ have been calculated in the L-MOKE geometry. These values are defined as follows: $R_{\mathrm{ss}(\mathrm{pp})}=r_{\mathrm{ss}(\mathrm{pp})} r_{\mathrm{ss}(\mathrm{pp})}^{*}$ and $R_{\mathrm{ps}(\mathrm{sp})}=r_{\mathrm{ps}(\mathrm{sp})} r_{\mathrm{ps}(\mathrm{sp})}^{*}$. The Fresnel coefficients $r_{\mathrm{ss}}, r_{\mathrm{pp}}, r_{\mathrm{ps}}$ and $r_{\mathrm{sp}}$ have been calculated with the aid of CST by calculating the scattering matrix elements in the defined waveguide port above the simulated structure. The simulating method is based on the calculation of the power of the electromagnetic waves impinging on the defined waveguide port (see ESI $\uparrow$ for further details), and provides very useful qualitative information about the reflectivity and the polarization conversion efficiency.

The simulated $R_{\mathrm{ss}}$ and $R_{\mathrm{pp}}$ for the corresponding oblique angle of incidence of $\theta_{\mathrm{i}}=55 \mathrm{deg}$, are presented in Fig. 3(a). For incident s-polarized light $\left(R_{\mathrm{ss}}\right)$, in the case of the film containing AuNPs, the characteristic minimum in the reflectivity associated with plasmon excitation is observed at $\sim 700 \mathrm{~nm}$. Plasmon excitation is further verified by the spectral position of the maximum of the near-field $\left\langle E_{y}\right\rangle$ (Fig. 2(a)), which is also very close to the minimum of the measured reflectance spectrum at $\sim 675 \mathrm{~nm}$. Furthermore, the simulated $R_{\mathrm{ss}}$ plot reproduces the corresponding experimental one, also very well. In the case of incident p-polarized light $\left(R_{\mathrm{pp}}\right)$, the modification due to the LPRs is stronger than in the case of incident s-polarized, as observed in the experimental $R_{\mathrm{pp}}$ curve in Fig. 1(c). It is worthwhile to notice that the influence of the two resonances associated to $\left\langle E_{x}\right\rangle$ and $\left\langle E_{z}\right\rangle$ appearing at two distinct spectral positions (see in Fig. 2(b)), becomes visible in the $R_{\mathrm{pp}}$ curve. This distinction is not clear in the experimental curves, we surmise, due to the dispersion of the aspect ratios of the AuNPs and other typical imperfections in the actual samples. To account for this distribution of the sizes and the aspect ratios, a large number of simulations over different configurations would be required, leading to an unaffordable timescale for each numerical spectrum.

In Fig. 3(b), we show the simulated polarization conversion efficiencies $R_{\mathrm{ps}}$ or $R_{\mathrm{sp}}$ for each sample. The polarization conversion efficiency expresses quantitatively the part of the intensity of the incident light which is converted from one polarization state to the other, upon reflection from the sample. These appear always to be identical in every case, namely $R_{\mathrm{ps}}=$ $R_{\mathrm{sp}}$. The latter is dictated by the symmetry imposed by the
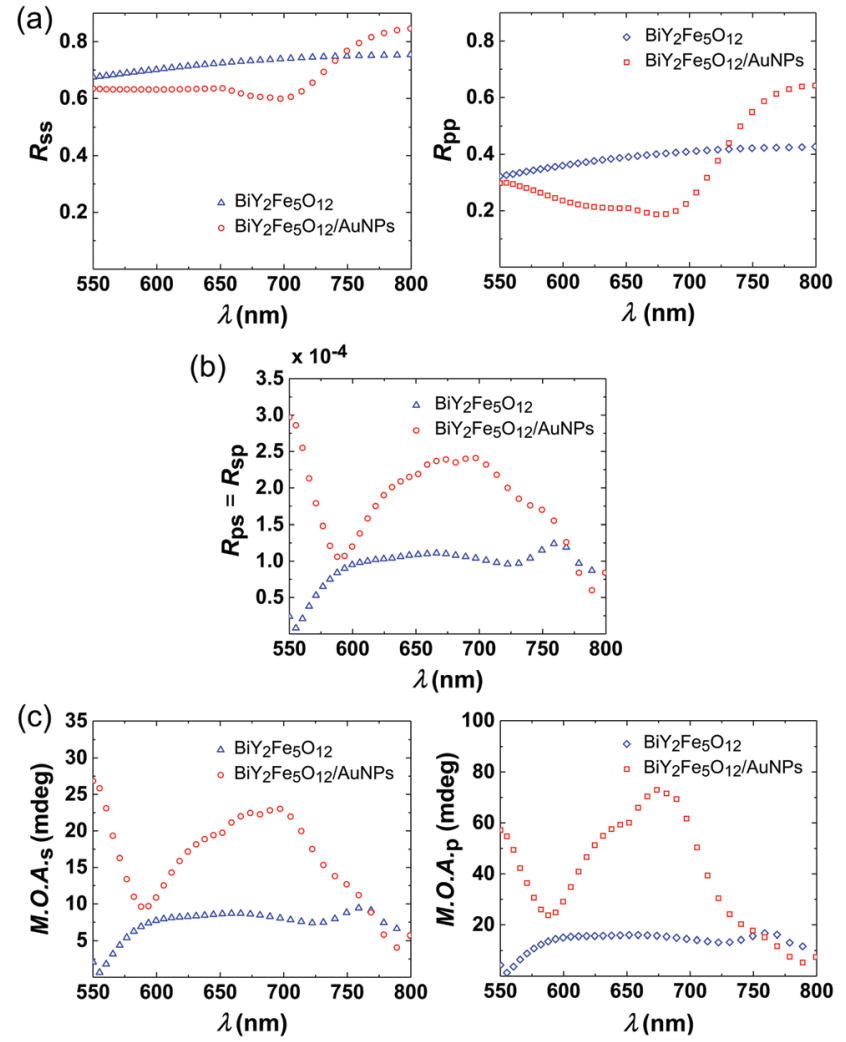

Fig. 3 Far-field simulations: (a) Simulated $R_{\mathrm{ss}}$ and $R_{\mathrm{pp}}$ for both samples. (b) Polarization conversion efficiency $R_{\mathrm{ps}}$ or $R_{\mathrm{sp}}$. (c) Post-calculated M.O.A., extracted from the simulated $r_{\mathrm{ss}}, r_{\mathrm{pp}}, r_{\mathrm{ps}}$ and $r_{\mathrm{sp}}$ values.

material itself $\left(\epsilon_{y z}=-\epsilon_{z y}\right)$. The simulations show that $R_{\mathrm{ps}(\mathrm{sp})}$ clearly exhibits a broadband enhancement in the spectral region of $600-800 \mathrm{~nm}$ where the plasmonic resonances are located, and it is independent of the incident light polarization. This proves that even in the case of incident s-polarized light, there is a resonance along the $z$ direction. This resonance is the result of polarization coupling, and it is mediated through the magneto-optical properties of Bi:YIG, giving rise to the broadband enhancement of the polarization conversion..$^{40,41}$ Indeed, if we express the complex oscillating electric dipole in the AuNP as $\boldsymbol{p}=\boldsymbol{\alpha}^{\mathrm{Au}} \boldsymbol{E}^{\mathrm{i}}$, where $\boldsymbol{E}^{\mathrm{i}}$ stands for the electric field of the incident light, then it can be shown that in linear response of $\epsilon_{y z}$ :

$$
\boldsymbol{\alpha}^{\mathrm{Au}}=\left(\begin{array}{ccc}
\alpha_{x x} & 0 & 0 \\
0 & \alpha_{y y} & \alpha_{y z} \\
0 & -\alpha_{y z} & \alpha_{z z}
\end{array}\right)
$$

where

$$
\alpha_{i i}=\frac{\left(\epsilon^{\mathrm{Au}}-\epsilon^{\mathrm{Bi} Y \mathrm{YIG}}\right) L_{\mathrm{i}}^{-1}}{\epsilon^{\mathrm{Au}}+\left(L_{\mathrm{i}}^{-1}-1\right) \epsilon^{\mathrm{Bi}: \mathrm{YIG}}} V \quad \text { with } i=x, y, z
$$

and

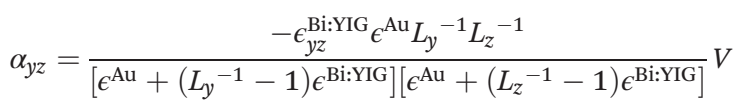


In eqn (6) and (7), $V$ is the volume of the nanoparticle, $L_{i}$ is the depolarizing factor along the $i^{t h}$, direction with $L_{x}+L_{y}+L_{z}=1$. In our case $L_{x}=L_{y}$, thus $\alpha_{x x}=\alpha_{y y}$ and different from $\alpha_{z z}$. The reader is referred to ESI $\dagger$ for further details on the derivation of eqn (6) and (7). As it can be observed from eqn (7), the offdiagonal element $\alpha_{y z}$ of the polarizability tensor of the AuNP is proportional to the off-diagonal element $\epsilon_{y z}^{\mathrm{Bi} Y \mathrm{YIG}}$. The latter, in terms of the damped harmonic oscillator model, implies that the directly generated electric dipole along the $y$ direction induces an indirect orthogonal electric dipole along the $z$ direction. Conceptually, the coupling between the two orthogonal electrical dipoles in the AuNP is mediated by the magneto-optically active Bi:YIG matrix, and is mathematically described by the non-zero $\epsilon_{y z}$ value. The concept of the coupled orthogonal LPRs has been experimentally demonstrated and described analytically by Lodewijks et al. and Maccaferri et al., ${ }^{24,42}$ only for circular and elliptical ferromagnetic islands, however. Quite differently here, we show the existence of coupled orthogonal LPRs in non-magnetic plasmonic nanoparticles $(\mathrm{Au})$, where the coupling is mediated by a magneto-optically active matrix. In Fig. 3(c), the post-calculated M.O.A. for the case of s- and p-polarized incident light are presented. The simulated M.O.A. reproduces very well the experimental one in the spectral region of about $675 \mathrm{~nm}$, in the case of the structure containing AuNPs. By comparing the simulated M.O.A. curves, we see that the one corresponding to the pure Bi:YIG structure without AuNPs, becomes largely modified by the presence of AuNPs. In the case of incident p-polarized light, the influence of the two characteristic features attributed to the LPRs along the two semi-axes of the nanoparticle, are visible. The distinction between these two resonances in the experimental data (Fig. 1(c)) are, again, washed out due to the distribution of the nanoparticle sizes and their aspect ratios. The modified M.O.A. in the far-field is generated by the landscape of the electric near-field modifications. These near-field features in the plasmonic structure have a direct impact on the exhibited reflectances as well as on the polarization conversion efficiency, and therefore on the magneto-optical activity.

\section{Conclusions}

In summary, we have experimentally demonstrated a broadband enhancement of the magneto-optical activity of hybrid Bi:YIG/AuNPs systems induced by localized plasmon resonances, by analyzing the longitudinal magneto-optical Kerr configuration. In order to unravel the role played by the localized plasmon resonances on the magneto-optical behaviour of the host Bi:YIG, we performed near-field simulations, and correlated the obtained results with the numerical far-field spectra. We have unambiguously shown that the features in the enhanced magneto-optical activity are the result of two orthogonal localized plasmon resonances that are coupled by the magneto-optical activity of the underlying Bi:YIG matrix, and are presented for both polarization states of the incident light. Our results pave the way to the design on-demand of the magneto-optical response of hybrid magneto-plasmonic circuitry, by controlling the localized resonances through the size and the aspect ratio of the nanoparticles.

\section{Experimental}

The Kerr rotation $(\theta)$ spectra measured with the aid of a spectroscopic Kerr effect setup operating in the longitudinal geometry (L-MOKE). A Wollaston prism, combined with a balanced photodiode setup mounted on a motorized rotational stage, was used to detect the rotation of the polarization axis. The Kerr ellipticity $(\epsilon)$ has been recorded as a function of wavelength, by incorporating into the detection path a superachromatic quarter-wave prism from Thorlabs. The setup is fully automated and, with proper calibration, can provide Kerr rotation and ellipticity in absolute values. The probing light in the range of 550-800 $\mathrm{nm}$ was produced by a supercontinuum laser light source (SuperK Extreme) and was properly monochromatized by an acousto-optical tunable filter. The reader is referred to the $\mathrm{ESI} \dagger$ for an indicative example of Kerr rotation and ellipticity measurements.

The specular reflectance spectra were recorded with the aid of the same setup, by replacing the balanced photodiode module with an integrating sphere photodiode power sensor. The power sensor was read by a PM400 power and energy meter from Thorlabs. An aluminium mirror was used as a reference for all of the recorded reflectance spectra.

\section{Conflicts of interest}

There are no conflicts to declare.

\section{Acknowledgements}

This work is funded by the Deutsche Forschungsgemeinschaft (DFG, German Research Foundation) - TRR 173 268565370. E. Th. P. and S. D. P. acknowledge the Carl Zeiss Foundation. Ken-ichi Uchida (NIMS, Japan) is acknowledged for providing the Bi:YIG/AuNPs samples. Dipl.-Phys. Marc Vogel - AG von Freymann - TU Kaiserslautern is acknowledged for the introduction to CST software and his help with the installation of the package.

\section{References}

1 G. Armelles, A. Cebollada, A. García-Martín and M. U. Gonzalez, Adv. Opt. Mater., 2013, 1, 10-35.

2 D. O. Ignatyeva, G. A. Knyazev, P. O. Kapralov, G. Dietler, S. K. Sekatskii and V. I. Belotelov, Sci. Rep., 2016, 6, 28077.

3 N. Maccaferri, E. K. Gregorczyk, T. V. A. G. de Oliveira, M. Kataja, S. van Dijken, Z. Pirzadeh, A. Dmitriev, 
J. Åkerman, M. Knez and P. Vavassori, Nat. Commun., 2015, 6, 6150 .

4 B. Caballero, A. García-Martín and J. C. Cuevas, ACS Photonics, 2016, 3, 203.

5 V. V. Temnov, G. Armelles, U. Woggon, D. Guzatov, A. Cebollada, A. García-Martín, J.-M. Garcia-Martin, T. Thomay, A. Leitenstorfer and R. Bratschitsch, Nat. Photonics, 2010, 4, 107.

6 K. Uchida, H. Adachi, D. Kikuchi, S. Ito, Z. Qiu, S. Maekawa and E. Saitoh, Nat. Commun., 2015, 6, 5910.

7 S. Ishii, K.-i. Uchida, T. D. Dao, Y. Wada, E. Saitoh and T. Nagao, APL Photonics, 2017, 2, 106103.

8 C. J. Firby and A. Y. Elezzabi, Appl. Phys. Lett., 2016, 109, 011101.

9 B. Sepúlveda, J. B. González-Díaz, A. García-Martín, L. M. Lechuga and G. Armelles, Phys. Rev. Lett., 2010, 104, 147401.

10 J. F. Torrado, E. T. Papaioannou, G. Ctistis, P. Patoka, M. Giersig, G. Armelles and A. García-Martín, Phys. Status Solidi RRL, 2010, 4, 271.

11 N. Maccaferri, X. Inchausti, A. García-Martín, J. C. Cuevas, D. Tripathy, A. O. Adeyeye and P. Vavassori, ACS Photonics, 2015, 2, 1769.

12 E. T. Papaioannou, T. Meyer and B. Hillebrands, J. Surf. Interfaces Mater., 2014, 2, 40-45.

13 I. Razdolski, D. G. Gheorghe, E. Melander, B. Hjörvarsson, P. Patoka, A. V. Kimel, A. Kirilyuk, E. T. Papaioannou and T. Rasing, Phys. Rev. B: Condens. Matter Mater. Phys., 2013, 88, 075436.

14 G. Armelles, A. Cebollada, F. García, A. García-Martín and N. de Sousa, ACS Photonics, 2016, 3, 2427.

15 B. Caballero, A. García-Martín and J. C. Cuevas, Opt. Express, 2015, 23, 22238.

16 D. Martin-Becerra, J. B. Gonzalez-Diaz, V. V. Temnov, A. Cebollada, G. Armelles, T. Thomay, A. Leitenstorfer, R. Bratschitsch, A. García-Martín and M. U. Gonzalez, Appl. Phys. Lett., 2010, 97, 183114.

17 H. Fang, B. Caballero, E. M. Akinoglu, E. T. Papaioannou, A. García-Martín, J. C. Cuevas, M. Giersig and P. Fumagalli, Appl. Phys. Lett., 2015, 106, 153104.

18 M. Kataja, T. K. Hakala, A. Julku, M. J. Huttunen, S. van Dijken and P. Torma, Nat. Commun., 2015, 6, 7072.

19 E. Melander, E. Östman, J. Keller, J. Schmidt, E. T. Papaioannou, V. Kapaklis, U. B. Arnalds, B. Caballero, A. García-Martín, J. C. Cuevas and B. Hjörvarsson, Appl. Phys. Lett., 2012, 101, 063107.

20 L. Chen, J. Gao, W. Xia, S. Zhang, S. Tang, W. Zhang, D. Li, X. Wu and Y. Du, Phys. Rev. B: Condens. Matter Mater. Phys., 2016, 93, 214411.

21 V. Bonanni, S. Bonetti, T. Pakizeh, Z. Pirzadeh, J. Chen, J. Nogués, P. Vavassori, R. Hillenbrand, J. Åkerman and A. Dmitriev, Nano Lett., 2011, 11, 5333.

22 E. T. Papaioannou, V. Kapaklis, E. Melander, B. Hjörvarsson, S. D. Pappas, P. Patoka, M. Giersig,
P. Fumagalli, A. García-Martín and G. Ctistis, Opt. Express, 2011, 19, 23867.

23 H. M. Luong, B. Ai, Y. Zhao and T. D. Nguyen, J. Magn. Magn. Mater., 2018, 468, 79.

24 N. Maccaferri, A. Berger, S. Bonetti, V. Bonanni, M. Kataja, Q. H. Qin, S. van Dijken, Z. Pirzadeh, A. Dmitriev, J. Noguós, J. Åkerman and P. Vavassori, Phys. Rev. Lett., 2013, 111, 167401.

25 V. I. Belotelov, I. A. Akimov, M. Pohl, V. A. Kotov, S. Kasture, A. S. Vengurlekar, A. V. Gopal, D. R. Yakovlev, A. K. Zvezdin and M. Bayer, Nat. Nanotechnol., 2011, 6, 370.

26 S. Tomita, T. Kato, S. Tsunashima, S. Iwata, M. Fujii and S. Hayashi, Phys. Rev. Lett., 2006, 96, 167402.

27 M. Rollinger, P. Thielen, E. Melander, E. Östman, V. Kapaklis, B. Obry, M. Cinchetti, A. García-Martín, M. Aeschlimann and E. T. Papaioannou, Nano Lett., 2016, 16, 2432.

28 T. A. Kelf, Y. Sugawara, R. M. Cole, J. J. Baumberg, M. E. Abdelsalam, S. Cintra, S. Mahajan, A. E. Russell and P. N. Bartlett, Phys. Rev. B: Condens. Matter Mater. Phys., 2006, 74, 245415.

29 E. T. Papaioannou, H. Fang, B. Caballero, E. M. Akinoglu, M. Giersig, A. García-Martín and P. Fumagalli, Opt. Express, 2017, 25, 32792.

30 O. Borovkova, A. Kalish and V. Belotelov, Opt. Lett., 2016, 41, 4593.

31 I. S. Maksymov, J. Hutomo and M. Kostylev, Opt. Express, 2014, 22, 8720.

32 E. Almpanis, P. Pantazopoulos, N. Papanikolaou, V. Yannopapas and N. Stefanou, J. Opt. Soc. Am. B, 2016, 33, 2609.

33 H. Uchida, Y. Masuda, R. Fujikawa, A. Baryshev and M. Inoue, J. Magn. Magn. Mater., 2009, 321, 843.

34 V. Doormann, J. P. Krumme, C. P. Klages and M. Erman, Appl. Phys. A, 1984, 34, 223.

35 S. Wittekoek, T. J. A. Popma, J. M. Robertson and P. F. Bongers, Phys. Rev. B: Solid State, 1975, 12, 2777.

36 V. Doormann, J. Krumme and H. Lenz, J. Appl. Phys., 1990, 68, 3544 .

37 A. D. Rakić, A. B. Djurišić, J. M. Elazar and M. L. Majewski, Appl. Opt., 1998, 37, 5271.

38 E. D. Palik and G. Ghosh, Handbook of optical constants of solids, Academic Press, San Diego, 1998.

39 V. G. Andrianova, V. S. Dozhdikov, O. Yu. Obukhov, V. A. Petrov, V. Yu. Reznik and O. N. Sevryukov, Teplofiz. Vys. Temp., 1983, 21(4), 680; High Temperature, 1983, 21(4), 513.

40 N. de Sousa, L. S. Froufe-Pérez, J. J. Sáenz and A. GarcíaMartín, Sci. Rep., 2016, 6, 30803.

41 S. Albaladejo, R. Gómez-Medina, L. S. Froufe-Pérez, H. Marinchio, R. Carminati, J. F. Torrado, G. Armelles, A. García-Martín and J. J. Sáenz, Opt. Express, 2010, 18, 3556.

42 K. Lodewijks, N. Maccaferri, T. Pakizeh, R. K. Dumas, I. Zubritskaya, J. Åkerman, P. Vavassori and A. Dmitriev, Nano Lett., 2014, 14, 7207. 